TITLE:

\title{
Dynamic polarization filtering in anisotropically strained M-plane GaN films
}

\section{AUTHOR(S):}

Omae, K; Flissikowski, T; Misra, P; Brandt, O; Grahn, HT; Kojima, K; Kawakami, Y

\section{CITATION:}

Omae, K ... [et al]. Dynamic polarization filtering in anisotropically strained M-plane GaN films. APPLIED PHYSICS LETTERS 2005, 86(19): 191909.

\section{ISSUE DATE:}

2005-05-09

URL:

http://hdl.handle.net/2433/50147

\section{RIGHT:}

Copyright 2005 American Institute of Physics. This article may be downloaded for personal use only. Any other use requires prior permission of the author and the American Institute of Physics. 


\title{
Dynamic polarization filtering in anisotropically strained M-plane GaN films
}

\author{
K. Omae, T. Flissikowski, P. Misra, O. Brandt, and H. T. Grahn ${ }^{a)}$ \\ Paul-Drude-Institut für Festkörperelektronik, Hausvogteiplatz, 5-7, 10117 Berlin, Germany \\ K. Kojima and Y. Kawakami \\ Department of Electronic Science and Engineering, Kyoto University, Kyoto 615-8510, Japan
}

(Received 15 February 2005; accepted 25 March 2005; published online 3 May 2005)

\begin{abstract}
The dynamic optical polarization filtering for anisotropically strained $M$-plane $\mathrm{GaN}$ films on $\mathrm{LiAlO}_{2}$ is investigated by pump and probe spectroscopy. Due to a very large polarization anisotropy in the absorption coefficient, these films exhibit an intrinsic polarization filtering, which appears as a rotation of the polarization vector after transmission of linearly polarized light through the film. For dynamic filtering, the polarization rotation is controlled by the pump, since the photoexcited carriers remove the intrinsic linear dichroism by selective bleaching of the anisotropic absorption. The dynamic behavior of the polarization rotation is mainly determined by the redistribution of holes between the two uppermost valence bands and by the recombination time. The latter is comparable to the measured decay time of the dynamic rotation of about $15 \mathrm{ps}$. For $M$-plane GaN films, the maximum dynamic rotation reaches $35^{\circ}$, while the maximum static polarization rotation is about 40․ () 2005 American Institute of Physics. [DOI: 10.1063/1.1927271]
\end{abstract}

Optical polarization anisotropy in semiconductors can be used to realize an optical modulator, which has both a high contrast and a fast response. ${ }^{1,2}$ Linear dichroism due to inherent or strain-induced anisotropies in the valence band (VB) structure gives rise to polarization filtering, which appears as a rotation of the polarization vector after the transmission of linearly polarized light. ${ }^{1,3}$ The polarization rotation can be modulated by selective photoexcitation, since the photoexcited carriers bleach the anisotropic absorption and remove the static linear dichroism.

Optical modulators based on polarization anisotropy can be realized using anisotropically strained films, e.g., $\mathrm{GaAs} /(\mathrm{Al}, \mathrm{Ga})$ As multiple quantum wells, where the typical time response is $0.5 \mathrm{ps}{ }^{1,4}$ For wurtzite semiconductors, the linear dichroism due to an in-plane polarization anisotropy is inherently present for $A$-plane $\mathrm{ZnO}$ films on $R$-plane sapphire ${ }^{5}$ and $M$-plane GaN films on $\mathrm{LiAlO}_{2}$ (Ref. 3) under normal incidence and is even enhanced for anisotropic strain. For $A$-plane $\mathrm{ZnO}$, the maximum reported values for the observed static and dynamic rotation are about $10^{\circ}$ and $12^{\circ}$, respectively. ${ }^{5}$ In contrast, $M$-plane $\mathrm{GaN}$ films on $\mathrm{LiAlO}_{2}$ exhibit a much larger in-plane optical anisotropy due to very large values of the components of the anisotropic in-plane strain. ${ }^{3}$ For a particular range of strain values, the wave function of the highest (second highest) VB becomes completely $|x\rangle(|z\rangle)$ like, ${ }^{6}$ which is very different from the wave function symmetry in unstrained and isotropically strained $C$-plane $\mathrm{GaN}$. As a result, $M$-plane $\mathrm{GaN}$ films on $\mathrm{LiAlO}_{2}$ exhibit a much larger absorption coefficient $\alpha$ within a certain energy range for the polarization $\mathbf{E} \perp \mathbf{c}$ than for $\mathbf{E} \| \mathbf{c}{ }^{7}$

In this letter, we investigate the dynamic polarization filtering for an anisotropically strained $M$-plane $\mathrm{GaN}$ film on $\mathrm{LiAlO}_{2}$, where the $c$ axis of the wurtzite crystal structure lies in the film plane. The observed static and dynamic polarization rotation in $M$-plane $\mathrm{GaN}$ films is much larger than for $A$-plane $\mathrm{ZnO}$ films on $R$-plane sapphire.
The $M$-plane GaN film was grown by rf plasma-assisted molecular-beam epitaxy on a $\gamma$ - $\mathrm{LiAlO}_{2}(100)$ substrate. ${ }^{8,9}$ The film thickness $d=0.7 \mu \mathrm{m}$ was determined by scanning electron microscopy. The film exhibits $n$-type conductivity with a background carrier density of about $10^{18} \mathrm{~cm}^{-3}$. To investigate the dynamic behavior of the polarization filtering, we performed time-resolved transmittance spectroscopy using the degenerate pump-and-probe technique. The pump and probe were adjusted to $360 \mathrm{~nm}(3.44 \mathrm{eV})$ using the second harmonic of a femtosecond Ti:Sapphire laser, since the film shows the largest difference of the absorption coefficients $\alpha_{\perp}-\alpha_{\|}$at this wavelength. Figure 1 displays a schematic diagram of the experimental setup and the definition of the coordinate system. The pump and probe beams are separated by the beam splitter. The ratio of the respective intensities is larger than 20:1 to avoid any influence of the probe beam on the optical polarization properties. The polarization of the pump and probe beams was independently controlled by two $\lambda / 2$ plates. Pump and probe beams are tightly focused onto the sample by a microscope objective to achieve a sufficiently high excitation intensity, which is estimated to be about $5 \mathrm{~mJ} / \mathrm{cm}^{2}$. In order to compensate for any birefringence by the substrate, we attached a second $\mathrm{LiAlO}_{2}$ substrate with the crystal orientation rotated by $90^{\circ}$. The ana-

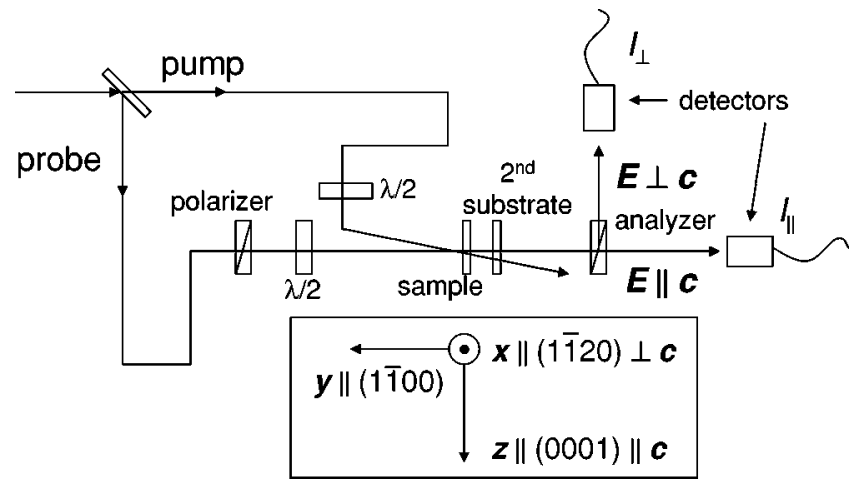

FIG. 1. Experimental setup and the definition of the coordinate system.

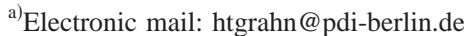




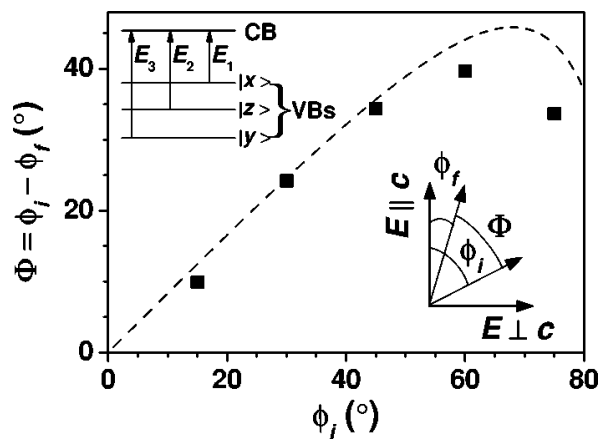

FIG. 2. Measured (squares) and calculated (dashed line) static rotation angle $\Phi$ for $T_{\perp} / T_{\|}=1 / 37 \mathrm{vs} \phi_{i}$ for $360 \mathrm{~nm}$ at $300 \mathrm{~K}$. The upper left inset connects the transitions $E_{1}, E_{2}$, and $E_{3}$ from the three uppermost valence bands (VBs) into the conduction band $(\mathrm{CB})$ to their respective polarizations $x, z$, and $y$. The lower right inset shows the definition of the angles and polarization directions.

lyzer after the sample is used to split the transmitted probe beam in two components $\mathbf{E} \| \mathbf{c}\left(\phi_{a}=0^{\circ}\right)$ and $\mathbf{E} \perp \mathbf{c}\left(\phi_{a}\right.$ $=90^{\circ}$ ), which are simultaneously measured by two photodiodes connected to two lock-in amplifiers.

Under normal incidence, the light propagates in the $y$ direction. Therefore, only the highest and second highest VBs have to be considered, since their polarization is $x$ - and $z$-like, respectively, as indicated in the upper left inset of Fig. 2. The effectiveness of the filtering of the incoming polarization vector with an angle $\phi_{i}$ with respect to the outgoing polarization vector with an angle $\phi_{f}$ can be quantified by the difference $\Phi$ as

$$
\Phi=\phi_{i}-\phi_{f}=\phi_{i}-\arctan \left(\sqrt{\frac{I_{\perp}^{0}}{I_{\|}^{0}}}\right),
$$

where $I_{\|}^{0}$ and $I_{\perp}^{0}$ denote the transmitted intensities of the probe beam without the pump beam separated by the analyzer according to their polarization as shown in Fig. 1. The filtering appears as a rotation by $\Phi$ of the incoming polarization vector. The birefringence of the film can be neglected around $360 \mathrm{~nm}^{7}$ The squares in Fig. 2 indicate the measured polarization rotation for $360 \mathrm{~nm}$ at $300 \mathrm{~K}$. $\Phi$ can be also expressed by

$$
\Phi=\phi_{i}-\arctan \left(\tan \left(\phi_{i}\right) \sqrt{\frac{T_{\perp}}{T_{\|}}}\right),
$$

where $T_{\perp}$ and $T_{\|}$denote the corresponding transmittances. The dashed line in Fig. 2 indicates the calculated value of $\Phi$ using Eq. (2) with a value of $T_{\perp} / T_{\|}=1 / 37$, which was independently measured by detecting the transmittances without an analyzer for $\phi_{i}=0$ and $90^{\circ} .{ }^{3}$ In this case, the probe beam at $360 \mathrm{~nm}$ was derived from a Xe arc-lamp filtered by a $0.64 \mathrm{~m}$ monochromator. Its intensity is several orders of magnitude smaller than the one of the probe beam of the pulsed laser. Both, the squares and the dashed line, agree very well over the whole range of the polarization angle of the incoming light beam. From this comparison, we conclude that the intensity of the probe beam is sufficiently weak so that it does not have any influence on the optical polarization properties of the film. The maximum static rotation of $40^{\circ}$ is observed for $\phi_{i}=60^{\circ}$. Note that the maximum static rotation is solely determined by the value of the ratio $T_{\perp} / T_{\|}=a^{2}$, since $\Phi_{\max }=\arctan [(1-a) /(2 \sqrt{a})]$, which becomes about $46^{\circ}$ for $a^{2}=1 / 37$.

Downloaded 05 Mar 2008 to 130.54 .110 .22 . Redistribution subject

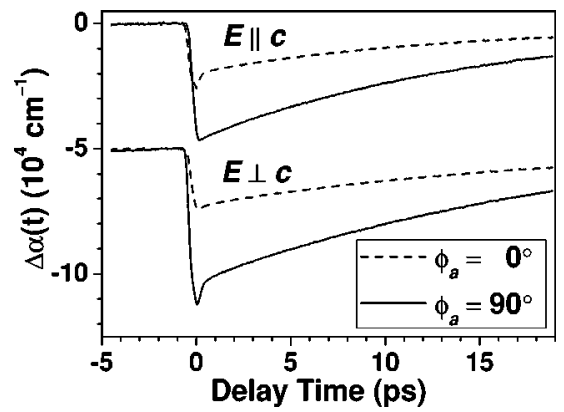

FIG. 3. Differential absorption spectra $\Delta \alpha(t)$ vs time delay for two pump polarizations $\mathbf{E} \| \mathbf{c}$ (upper curves) and $\mathbf{E} \perp \mathbf{c}$ (lower curves) and two analyzer angles $\phi_{a}=90^{\circ}$ (dashed lines) and $0^{\circ}$ (solid lines) recorded for $360 \mathrm{~nm}$ at $300 \mathrm{~K}$.

Figure 3 shows the differential absorption $\Delta \alpha$ versus time delay between pump and probe for $\phi_{i}=60^{\circ}$ for two pump polarization and two analyzer angles. The quantity $\Delta \alpha$ is related to the measured intensities by

$$
\Delta \alpha_{j}=\alpha_{j}-\alpha_{j}^{0}=\frac{1}{d} \ln \frac{I_{j}^{0}}{I_{j}},
$$

where $j$ denotes an analyzer angle of either $\phi_{a}=0^{\circ}(\|)$ or $\phi_{a}=90^{\circ}(\perp) . \alpha_{j}^{0}\left(I_{j}^{0}\right)$ and $\alpha_{j}\left(I_{j}\right)$ are the corresponding absorption coefficients (transmitted intensities) without and with the pump beam, respectively. $\Delta \alpha$ is modulated by the bleaching of the probe absorption due to the pump. When the pump beam has a perpendicular (parallel) polarization with respect to the $c$ axis, only the uppermost (second highest) VB is directly excited due to the polarization selection rules. If the relaxation of electrons within the conduction band and holes within the VB can be neglected, $\alpha_{\perp}(t)$ should only be modulated for a pump polarization $\mathbf{E} \perp \mathbf{c}$, while $\alpha_{\|}(t)$ should only be affected for $\mathbf{E} \| \mathbf{c}$. However, a strong modulation of both $\alpha_{\perp}$ and $\alpha_{\|}$is observed in Fig. 3 for both pump polarizations, indicating the redistribution of photoexcited holes between the two uppermost VBs. A fast initial decay of $\Delta \alpha$ with a time constant of about $200 \mathrm{fs}$, which is comparable to the time resolution of the laser system (mainly determined by the pulse width), is only observed in Fig. 3, when the analyzer is aligned parallel to the pump polarization. This initial decay is probably caused by the redistribution of holes between the uppermost and second highest VB. Ye et al. ${ }^{10}$ reported for $p$-type GaN that the hole-hole scattering time and the hothole cooling time are 50 and $600 \mathrm{fs}$, respectively. These values imply that the redistribution of the holes is probably mediated by hole-hole scattering. However, the actual value of the redistribution time cannot be determined with our current setup due to the time resolution of about $200 \mathrm{fs}$. In order to clarify the hole relaxation mechanism in the VBs, additional systematic investigations are necessary. The time constant of the slower decay is about 15 ps for all four curves shown in Fig. 3. This time is very similar to the recombination time of about $11 \mathrm{ps}$, which was independently determined by time-resolved photoluminescence spectroscopy. At $300 \mathrm{~K}$, the recombination process is dominated by nonradiative recombination. By introducing additional nonradiative recombination centers, the response time of the polarization modulator can be reduced.

Figure 4 shows the dynamic rotation $\Phi(t)$, which is determined from the transmitted probe intensities $I_{\perp}(t)$ and $I_{\|}(t)$ using Eq. (1), as a function of time delay for two pump
AIP license or copyright; see http://apl.aip.orglapl/copyright.jsp 


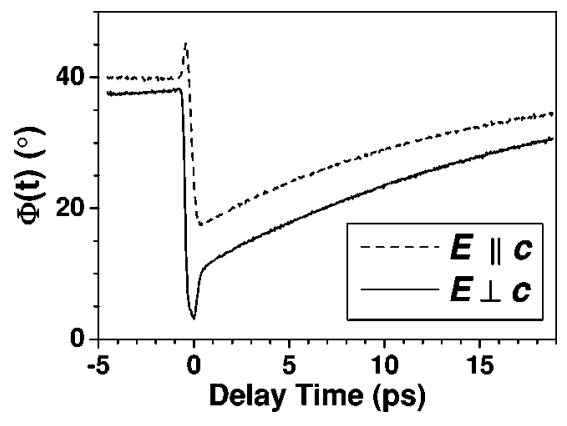

FIG. 4. Dynamic rotation $\Phi(t)$ vs time delay for two pump polarizations $\mathbf{E} \| \mathbf{c}$ (dashed line) and $\mathbf{E} \perp \mathbf{c}$ (solid line) recorded for $\phi_{i}=60^{\circ}$ at $300 \mathrm{~K}$.

polarizations at $\phi_{i}=60^{\circ}$ and $300 \mathrm{~K}$. The initial response decays very rapidly due to the hole redistribution between the two uppermost VBs. After the hole redistribution is completed within less than $1 \mathrm{ps},\left|\Phi_{\perp}(t)-\Phi_{\perp}^{0}\right|$ is only about $20 \%$ larger than $\left|\Phi_{\|}(t)-\Phi_{\|}^{0}\right|$, where $\Phi_{j}^{0}$ denotes the static rotation angle. While the average value of $\Phi_{\perp}(t)$ and $\Phi_{\|}(t)$ is determined by the redistribution time constants of the holes between the two uppermost VBs, the difference between $\Phi_{\perp}(t)$ and $\Phi_{\|}(t)$, which is almost time independent, originates from the different density of states of the two uppermost VB states. The actual value of $\Phi(t)$ strongly depends on the pump intensity. From the excitation density dependence of the rotation angle recorded at $1 \mathrm{ps}$, the excited carrier density for the pump polarization $\mathbf{E} \| \mathbf{c}$ can be estimated to be about $40 \%$ of the density for $\mathbf{E} \perp \mathbf{c}$. Note that the initial response of $\Phi(t)$ points in opposite directions for the two different polarization directions of the pump. Since the intensities for both analyzer angles are recorded simultaneously, the rise time of $I_{\|}$and $I_{\perp}$ for a given pump polarization can be directly compared. It turns out that the rise time of $I_{\|}$is faster than the one of $I_{\perp}$, when the pump polarization is parallel to the $c$ axis (not shown).

We also measured the dynamic rotation as a function of the polarization angle of the incoming light. For all initial polarization angles of the probe beam, $\Phi(t=0)$ for the pump polarized $\mathbf{E} \perp \mathbf{c}$ is less than $6^{\circ}$, since the static rotation is almost compensated by the bleaching of the absorption due to the photoexcited carriers. The maximum change in the dynamic rotation, which is determined by the absolute value of the difference between the value of the dynamic rotation at $t=0$ and the static rotation of the probe beam $(\mid \Phi(t=0)$ $\left.-\Phi^{0} \mid\right)$, becomes largest for $\phi_{i}=60^{\circ}$ with a value of $35^{\circ}$. Since the bleaching almost removes the static anisotropy of the absorption coefficient, the maximum dynamic rotation occurs for the same value of $\phi_{i}$ as the maximum static rotation.

In summary, for an $M$-plane GaN film on $\mathrm{LiAlO}_{2}$, a static and a dynamic rotation angle of $40^{\circ}$ and $35^{\circ}$, respectively, can be realized at $300 \mathrm{~K}$, which is observed for a polarization angle of the incoming light of $\phi_{i}=60^{\circ}$. Note that these values are much larger than the reported values for $A$-plane $\mathrm{ZnO}$ on $R$-plane sapphire. ${ }^{5}$ At zero time delay, the static optical anisotropy of the absorption coefficient is almost removed by the bleaching of the absorption due to the photoexcited carriers of the pump. The temporal behavior of the polarization rotation is mainly determined by the redistribution of holes between the two uppermost valence bands and the recombination time. At room temperature, the response time of the modulator is basically determined by the nonradiative recombination time.

One of the authors (K. O.) acknowledges financial support through a Research Fellowship for Young Scientists by the Japan Society of the Promotion of Science.

${ }^{1}$ H. Shen, M. Wraback, J. Pamulapati, M. Dutta, P. G. Newman, A. Ballato, and Y. Lu, Appl. Phys. Lett. 62, 2908 (1993).

${ }^{2}$ D. S. McCallum, X. R. Huang, A. L. Smirl, D. Sun, and E. Towe, Appl. Phys. Lett. 66, 2885 (1995).

${ }^{3}$ P. Misra, Y. Sun, O. Brandt, H. T. Grahn, and K. H. Ploog, Appl. Phys. Lett. 83, 4327 (2003).

${ }^{4}$ M. Wraback, H. Shen, J. Pamulapati, M. Dutta, P. G. Newman, M. Taysing-Lara, and Y. Lu, Surf. Sci. 305, 238 (1994).

${ }^{5}$ M. Wraback, H. Shen, S. Liang, C. R. Gorla, and Y. Lu, Appl. Phys. Lett. 74, 507 (1999).

${ }^{6}$ S. Ghosh, P. Waltereit, O. Brandt, H. T. Grahn, and K. H. Ploog, Phys. Rev. B 65, 075202 (2002).

${ }^{7}$ P. Misra, Y. Sun, O. Brandt, and H. T. Grahn, J. Appl. Phys. 96, 7029 (2004).

${ }^{8}$ P. Waltereit, O. Brandt, M. Ramsteiner, R. Uecker, P. Reiche, and K. H. Ploog, J. Cryst. Growth 218, 143 (2000).

${ }^{9}$ Y. J. Sun, O. Brandt, and K. H. Ploog, J. Vac. Sci. Technol. B 21, 1350 (2003).

${ }^{10}$ H. Ye, G. Wicks, and P. Fauchet, Appl. Phys. Lett. 77, 1185 (2000). 